\title{
Evolução do estado nutricional de menores de 5 anos em aldeias indígenas da Tribo Parakanã, na Amazônia Oriental Brasileira $(1989-1991)^{*}$
}

\author{
Nutritional status of Parakanã Indian - children from birth through 5 years of age, \\ eastern Amazonia, Brazil
}

\author{
Sandro J. Martins ${ }^{\star \star}$, Raimundo C. Menezes ${ }^{\star \star}$
}

\begin{abstract}
MARTINS, S.J. \& MENEZES, R.C. Evolução do estado nutricional de menores de 5 anos em aldeias indigenas da Tribo Parakanã, na Amazônia Oriental Brasileira (1989-1991). Rev. Saúde Pública, 28: 1-8, 1994. Foi estudada a recente evolução do estado nutricional de menores de 5 anos nas aldeias Parakanã, Maroxewara e Paranatinga. Dados antropométricos (peso, altura) foram obtidos em três estudos transversais para avaliação da prevalência de desnutrição e acompanhamento da evolução nutricional pelo incremento médio do indice peso/idade de 70 crianças ( $87,5 \%$ dos menores existentes). A desnutrição nas aldeias foi proporcional ao tempo de contato com nossa cultura e atribuivel a fatores socioculturais relatados. Na Paranatinga, os valores médios dos indicadores antropométricos peso/idade, altura/idade e peso/altura - foram os menores, principalmente nas crianças entre o sexto e o $24^{\circ}$ mês. Reduziu-se em $76,1 \%$ a prevalência de desnutrição global no grupo estudado, não havendo mais casos de desnutrição aguda na última avaliação. $O$ incremento do indicador peso/idade foi proporcional à severidade do caso, não havendo diferença significante entre as aldeias. Medidas de natureza preventiva adotadas foram eficazes em reduzir a prevalència de desnutrição infantil entre os Parakanã.
\end{abstract}

Descritores: Índios sul-americanos. Desnutrição protéico-calórica, epidemiologia. Antropometria, métodos.

\section{Introdução}

O crescimento e o desenvolvimento infantil são o núcleo integrador das ações de saúde materno-infantil e a base para as atividades de assistência primária de saúde; em última análise revelam em uma população a eficácia global das intervenções de saúde, tanto curativas como preventivas. A vigilância do processo de crescimento e desenvolvimento infantil se concebe como uma "medicina do desenvolvimento" que oferece ao individuo as máximas possibilidades de saúde e bem-estar ${ }^{3}$.

$O$ aumento mensal de peso é o indicador individual mais significativo do desenvolvimento normal da criança. Em paises em desenvolvimento, usa-se recorrer a agentes comunitários de saúde

* Trabalho realizado pelo Programa de Pesquisas em Saúde Indigena do Núcleo de Medicina Tropical da Universidade Federal do Pará, com apoio logístico do Programa Parakanà.

** Núcleo de Medicina Tropical da Universidade Federal do Pará - Belém, PA - Brasil

Separatas/Reprints: S.J. Martins - Av. Generalissimo Deodoro, 92 Unarizal - 66055-240 - Belém, PA - Brasil para ajudar as mães a pesarem regularmente todos os menores de 3 anos, o que ajuda os pais e os serviços de saúde a detectarem deficiências no desenvolvimento infantil? .

O diagnóstico de deficiência de crescimento por desnutrição, pela antropometria, baseia-se no encontro de medidas que, sendo suficientemente baixas, são de ocorrência improvável em indivíduos bem nutridos ${ }^{12}$. Por ser um método de baixo custo e boa significância, é apropriado para investigações em nivel primário, onde toda orientação do processo de investigação (pessoal, desenho do estudo, coleta de dados, métodos de análise e ações baseadas nos resultados) precisam manter-se dentro dos limites, recursos e capacidades locais de decisão $^{2,19}$.

O acompanhamento nutricional e a instituição de medidas de intervenção oportunas, que melhorem o estado de saúde e a nutrição, reduzem a influência de fatores ambientais que dificultam o alcance por parte das crianças de seu potencial genético de crescimento; tais medidas permitem que as crianças cresçam e se desenvolvam o mais próximo da forma com que crescem e se desenvol- 
vem as crianças sadias, em locais onde as familias dispõem de condições adequadas de saúde e alimentação ${ }^{2,8}$.

No Brasil vários estudos têm sido conduzidos com o objetivo de avaliar o estado nutricional infantil de populações de baixa renda, e alguns, menos recentes, abordam a questão em comunidades indígenas. As precárias condições de saúde dessas populações, em particular a elevada prevalência de doenças infecciosas, têm tido implicações com a prevalência de desnutrição infantil ${ }^{1,5,6,11,17,21}$.

O grupo indígena Parakanã habita a zona divisória entre as bacias dos rios Xingu e Tocantins, no sudeste do Estado do Pará, vivendo atualmente em três aldeamentos (Apyterewa, Maroxewara e Paranatinga) ${ }^{16}$.

Três fatos marcaram o encontro dos Parakanã com nossa cultura: na década de 1920 , a construção da Estrada de Ferro do Tocantins; no início da década de 70 , a construção da rodovia Transamazônica e, por último, nos anos 80 , a construção da Usina Hidrelétrica de Tucuruí (U.H.T.); em novembro de 1984 , com a inauguração da U.H.T., iniciouse o enchimento do lago que inundou 38.700 hectares de florestas em terras pertencentes aos Parakanã. A execução desses empreendimentos determinou deslocamentos involuntários dos grupos nativos, que repercutiram no modo de organização social e econômica dessas populações.

A partir de 1987, como forma de ressarcimento à comunidade Parakanã pelos danos causados pela U.H.T., as Centrais Elétricas do Norte do Brasil S.A. (ELETRONORTE) vem financiando o Programa Parakanã, gerenciado pela Fundação Nacional do Índio (FUNAI), que visa equilibrar as relações econômicas e culturais entre a sociedade Parakanã e a sociedade nacional além de garantir o usufruto exclusivo das terras demarcadas aos Parakanã. Para atingir essas metas, o Programa se divide em ações na área de saúde, educação, produção econômica e vigilância de limites. As aldeias Maroxewara e Paranatinga encontram-se na área da Reserva Indigena Parakanã, assistidas pelo Programa Parakanã. Desde o início de 1989, quando suas populações eram de 97 e 178 habitantes, respectivamente, as ações de saúde entre os Parakanã deixaram de ser executadas exclusivamente pela FUNAI, passando a ser desenvolvidas com ênfase nas atividades preventivas, sob orientação do Núcleo de
Medicina Tropical da Universidade Federal do Pará. Naquela época, um grave quadro de desnutrição afetava a aldeia Paranatinga ${ }^{14}$.

O presente trabalho tem por objetivo apresentar a evolução observada do estado nutricional dos menores de 5 anos existentes nas duas aldeias em abril de 1989, procurando caracterizar, naquela população, o impacto das medidas de prevenção primária e secundária adotadas sobre seu estado nutricional e a participação de fatores ambientais na gênese do processo de desnutrição.

\section{Metodologia}

A população em estudo foi constituída pela população de menores de 5 anos existente nas aldeias Maroxewara e Paranatinga, em abril de 1989, num total de 80 crianças. Naquela época, o grupo representava $27,9 \%$ da população nas duas aldeias: $32(31,1 \%)$ na Maroxewara e $48(26,1 \%)$ na Paranatinga; nos últimos 10 anos, todos os nascimentos tiveram seus registros efetuados em livros existentes nas próprias aldeias, de acordo com as normas da FUNAI.

O peso foi medido em quilogramas e a altura em centímetros, mediante o uso de balanças antropométricas. Para crianças menores, o comprimento foi medido por fita métrica não-distensivel em dispositivo convencional e, por razões logísticas, registrou-se o peso diferencial (mãe-filho). O desvio entre a medição antropométrica e a mediana correspondente da população de referência (valores do "National Center for Health Statistics - NCHS EUA) foi expresso em percentagem de adequação para o indicador peso/idade e em "Z-score" para os indicadores peso/idade, peso/altura e a altura/idade; estes valores foram obtidos por meio de programa para microcomputador desenvolvido pelo "Center for Disease Control" ${ }^{\prime 4}$.

As crianças que apresentaram adequação ponderal inferior a $90 \%$ dos valores medianos do padrão NCHS, suas nutrizes e as gestantes passaram a receber suplementação alimentar (proteinato de cálcio, amido de arroz para menores de 24 meses e outra fonte regional de carbohidratos para crianças maiores, gestantes e nutrizes). Em cada aldeia, uma enfermeira e uma técnica em enfermagem foram instruídas a prestar serviços de assistência curativa e preventiva (diagnóstico e tratamento de doenças 
respiratórias e diarréicas, curativos, vacinação, acompanhamento de partos), além do que realizavam sistematicamente pesquisa copro e hemoparasitológica (métodos de Hoffman e de Willis, gota espessa) e instituíam tratamento adequado a individuos parasitados. Um médico sanitarista orientava as atividades de campo das profissionais via rádio e em visitas à área.

$O$ estado nutricional foi avaliado através dos critérios propostos por Gomez, por Waterlow ${ }^{22}$ e pela Organização Mundial da Saúde (OMS) ${ }^{23}$, considerando como desnutridos quem apresentasse menos de $90 \%$ do peso mediano para sua idade e sexo (Gomez) ou se encontrasse com peso para altura, altura para idade ou peso para altura abaixo de -2 unidades de desvio-padrão em relação à mediana da população de referência ("Z-score") ${ }^{22,23}$. O termo "desnutrição global" foi empregado para descrever crianças com déficit de peso para idade; "desnutrição pregressa" refere-se aos déficits de altura para idade; "desnutrição aguda" aos déficits de peso para altura, e, "desnutrição crônica", aos déficits combinados de peso para altura e altura para idade.
A eficácia do programa de suplementação alimentar e do conjunto de medidas adotadas sobre o estado nutricional do grupo foi avaliada com base na observação da velocidade de crescimento das crianças no período, como proposto por Lei e col. ${ }^{9}$ (1989), pela observação do comportamento do índice de peso para a idade em três momentos: abril de 1989, janeiro de 1990 e outubro de 1991. Considerou-se como casos de sucesso os que apresentaram, na última avaliação, um incremento de "Z"maior que 0,55 , o suficiente para esperar-se uma redução de pelo menos $30 \%$ no déficit global de peso para a idade na população em estudo. Este valor foi estabelecido após a avaliação das primeiras medições, que davam conta que a média do índice peso/idade no grupo era de $-1,79 \pm 1,31$ Z-score.

Como teste de independência entre freqüências foi usado, a prova do qui-quadrado, com a correção de continuidade (Yates) sempre que $\mathrm{GL}=1$; para analisar a variância de sub-grupos pelos valores médios dos indicadores (peso/idade, altura/idade, pelo/altura e incremento de " $Z$ ") usou-se o teste de Kruskal-Wallis ${ }^{20}$. Em todos os casos foi

Tabela 1. Quadro nutricional da população Parakanā por aldeia, período de estudo e critério de avaliação.

\begin{tabular}{|c|c|c|c|c|c|c|c|c|c|}
\hline \multirow{2}{*}{$\begin{array}{l}\text { Indicador de } \\
\text { Desnutrição }\end{array}$} & \multicolumn{3}{|c|}{ Geral } & \multicolumn{3}{|c|}{ Paranatinga } & \multicolumn{3}{|c|}{ Maroxewara } \\
\hline & $04 / 89$ & $01 / 90$ & $10 / 91$ & $04 / 89$ & $01 / 90$ & $10 / 91$ & $04 / 89$ & $01 / 90$ & $10 / 91$ \\
\hline \multicolumn{10}{|l|}{ OMS (-2 Z-score) } \\
\hline Peso/idade & $42,3 \%$ & $19,0 \%$ & $10,1 \%$ & $63,0 \%$ & $26,1 \%$ & $17,4 \%$ & $4,0 \%$ & $9,1 \%$ & $\cdot$ \\
\hline Altura/idade & $53,5 \%$ & $48,1 \%$ & $50,6 \%$ & $78,3 \%$ & $71,7 \%$ & $73,9 \%$ & $8,0 \%$ & $15,2 \%$ & $18,2 \%$ \\
\hline Peso/altura & $11,3 \%$ & $1,3 \%$ & $\cdot$ & $13,0 \%$ & - & $\cdot$ & $8,0 \%$ & $3,0 \%$ & - \\
\hline \multicolumn{10}{|l|}{ Gomez } \\
\hline Sobrepeso & • & $1,3 \%$ & $1,3 \%$ & $\cdot$ & $2,2 \%$ & - & - & - & $3,0 \%$ \\
\hline Normal & $26,8 \%$ & $27,8 \%$ & $34,1 \%$ & $10,9 \%$ & $10,9 \%$ & $19,6 \%$ & $56,0 \%$ & $51,5 \%$ & $54,6 \%$ \\
\hline Grau I & $39,4 \%$ & $60,8 \%$ & $59,5 \%$ & $39,1 \%$ & $73,9 \%$ & $71,7 \%$ & $40,0 \%$ & $42,4 \%$ & $42,4 \%$ \\
\hline Grau II & $23,9 \%$ & $7,6 \%$ & $5,1 \%$ & $34,8 \%$ & $13,0 \%$ & $8,7 \%$ & $4,0 \%$ & - & - \\
\hline Grau III & $9,9 \%$ & $2,5 \%$ & - & $15,2 \%$ & - & • & • & $6,1 \%$ & $\cdot$ \\
\hline \multicolumn{10}{|l|}{ Waterlow } \\
\hline Eutrófico & $43,6 \%$ & $50,6 \%$ & $49,4 \%$ & $19,6 \%$ & $28,3 \%$ & $26,1 \%$ & $88,0 \%$ & $81,8 \%$ & $81,8 \%$ \\
\hline Desn. aguda & $2,8 \%$ & $1,3 \%$ & - & $2,1 \%$ & - & - & $4,0 \%$ & $3,0 \%$ & - \\
\hline Desn. crônica & $8,5 \%$ & - & $\cdot$ & $10,9 \%$ & - & $\cdot$ & $4,0 \%$ & • & $\cdot$ \\
\hline Desn. pregressa & $45,1 \%$ & $48,1 \%$ & $50,6 \%$ & $67,4 \%$ & $71,7 \%$ & $73,9 \%$ & $4,0 \%$ & $15,2 \%$ & $18.2 \%$ \\
\hline \multicolumn{10}{|l|}{$N^{2}$ de crianças } \\
\hline avaliadas & 71 & 79 & 79 & 46 & 46 & 46 & 25 & 33 & 33 \\
\hline
\end{tabular}


estabelecido $5 \%$ como nível de significância.

Todos os cálculos foram realizados em microcomputador, usando um programa para vigilância epidemiológica de domínio público (Epi Info, versão 5.0) e um gerenciador de bancos de dados.

\section{Resultados}

Foram incluídas no presente estudo as medidas tomadas em abril de 1989, janeiro de 1990 e outru- bro de 1991, pois foram as que incluiram a maior parte dos menores de 5 anos (respectivamente $88,7 \%, 98,7 \%$ e $98,7 \%) ; 70$ crianças $(87,5 \%$ do grupo em questão) foram acompanhadas nas três medições. Nenhuma das crianças avaliadas em abril de 1989 morreu no período do estudo.

As medidas de prevalência de desnutrição e a avaliação nutricional do grupo estudado encontram-se nas Tabelas 1 e 2 . Observa-se que no período operou-se uma redução da ordem de $76,1 \%$ (de

Tabela 2. Variabilidade (média \pm DP) dos indicadores antropométricos e do incremento médio do indicador peso/idade, por aldeia, sexo e idade.

\begin{tabular}{|c|c|c|c|c|c|}
\hline \multirow[b]{2}{*}{ Grupo } & \multirow[b]{2}{*}{ № } & \multicolumn{3}{|c|}{ Abril de 1989} & \multirow{2}{*}{$\begin{array}{l}\text { Incremento de "Z" } \\
\qquad(89 / 91) *\end{array}$} \\
\hline & & Peso/idade & Altura/idade & Peso/altura & \\
\hline \multicolumn{6}{|l|}{$\begin{array}{l}\text { Paranatinga } \\
\text { Idade (meses) }\end{array}$} \\
\hline 0.6 & 3 & $-0,90 \pm 0,44$ & $-0,30 \pm 0,92$ & $-0,80 \pm 1,31$ & $-0,37 \pm 0,55$ \\
\hline $6-12$ & 11 & $-3,48 \pm 0,93$ & $-3,15 \pm 1,45$ & $-1,47 \pm 1,31$ & $2,17 \pm 1,44$ \\
\hline $12-24$ & 9 & $-2,97 \pm 1,05$ & $-3,36 \pm 1,81$ & $-1,18 \pm 1,31$ & $1,68 \pm 1,08$ \\
\hline $24-36$ & 7 & $-2,21 \pm 0,57$ & $-2,61 \pm 0,69$ & $-0,80 \pm 1,31$ & $0,23 \pm 0,81$ \\
\hline $36 \cdot 48$ & 9 & $-1,52 \pm 0,73$ & $-2,34 \pm 0,71$ & $-0,17 \pm 1,31$ & $0,18 \pm 0,65$ \\
\hline $48-60$ & 7 & $-1,50 \pm 0,99$ & $-2,29 \pm 1,48$ & $-0,16 \pm 1,31$ & $0,09 \pm 0,38$ \\
\hline Diferença & & $p<0,00013$ & $p<0,049$ & $p<0,039$ & $p<0,00025$ \\
\hline \multicolumn{6}{|l|}{ Sexo } \\
\hline Masculino & 24 & $-1,99 \pm 1,09$ & $-2,33 \pm 1,04$ & $-0,66 \pm 1,13$ & $0,67 \pm 1,40$ \\
\hline Feminino & 22 & $-2,71 \pm 1,23$ & $-2,96 \pm 1,76$ & $-0,98 \pm 1,23$ & $1,11 \pm 1,20$ \\
\hline Diferença & & $p<0,029$ & N.S. & N.S. & N.S. \\
\hline \multicolumn{6}{|l|}{$\begin{array}{l}\text { Maroxewara } \\
\text { Idade (meses) }\end{array}$} \\
\hline 0.6 & 3 & $0,03 \pm 0,15$ & $0,53 \pm 0,76$ & $-0,50 \pm 0,62$ & $-0,83 \pm 0,85$ \\
\hline $6 \cdot 12$ & 4 & $-1,02 \pm 0,67$ & $-0,35 \pm 1,89$ & $-0,67 \pm 2,09$ & $0,40 \pm 0,76$ \\
\hline $12 \cdot 24$ & 4 & $-1,60 \pm 1,30$ & $-1,80 \pm 0,99$ & $-0,75 \pm 1,33$ & $0,85 \pm 0,77$ \\
\hline $24 \cdot 36$ & 6 & $-0,75 \pm 0,59$ & $-0,78 \pm 0,44$ & $-1,60 \pm 0,64$ & $0,32 \pm 0,35$ \\
\hline $36 \cdot 48$ & 4 & $-0,85 \pm 0,57$ & $-0,93 \pm 0,38$ & $-0,22 \pm 0,97$ & $1,28 \pm 2,16$ \\
\hline $48 \cdot 60$ & 4 & $-0,33 \pm 0,45$ & $-1,90 \pm 0,70$ & $1,13 \pm 0,88$ & $-0,33 \pm 0,36$ \\
\hline Diferença & & N.S. & $p<0,029$ & N.S. & N.S. \\
\hline \multicolumn{6}{|l|}{ Sexo } \\
\hline Masculino & 13 & $-0,94 \pm 0,99$ & $-1,11 \pm 1,38$ & $-0,28 \pm 1,47$ & $0,28 \pm 0,68$ \\
\hline Feminino & 12 & $-0,62 \pm 0,56$ & $-0,72 \pm 0,87$ & $-0,12 \pm 0,94$ & $0,37 \pm 1,50$ \\
\hline Diferença & & N.S. & N.S. & N.S. & N.S. \\
\hline \multicolumn{6}{|l|}{ Geral } \\
\hline Paranatinga & 46 & $-2,33 \pm 1,20$ & $-2,63 \pm 1,45$ & $-0,81 \pm 1,17$ & $0,88 \pm 1,31$ \\
\hline Maroxewara & 25 & $-0,79 \pm 0,81$ & $-0,91 \pm 1,16$ & $-0,21 \pm 1,22$ & $0,32 \pm 1,12$ \\
\hline Diferença & & $p<0,000002$ & $p<0,000002$ & $p<0,018$ & N.S. \\
\hline
\end{tabular}

- Os valores referentes à aldeia Paranatinga correspondem aos das 45 crianças que foram avaliadas em abril de 1989 e outubro de 1991. 
Tabela 3. Quadro evolutivo do estado nutricional de 70 crianças, avaliadas em abril de 89 e outubro de 91 , por aldeia.

\begin{tabular}{|c|c|c|c|c|}
\hline \multirow{2}{*}{ Aldeia } & \multicolumn{3}{|c|}{ Classe nutricional } & \multirow{2}{*}{ Diferença* } \\
\hline & Normal & Grau 1 & Graus $\|$ e II & \\
\hline \multicolumn{5}{|l|}{ Paranatinga } \\
\hline No crianças & 5 & 18 & 22 & - \\
\hline$\%$ Sucesso ** & 20,0 & 22,2 & 77,3 & $p<0,001$ \\
\hline Incremento & $-0,25 \pm 0,61$ & $0,18 \pm 0,63$ & $1,70 \pm 1,34$ & $p<0,00006$ \\
\hline \multicolumn{5}{|l|}{ Maroxewara } \\
\hline $\mathrm{N}^{2}$ crianças & 14 & 10 & 1 & - \\
\hline \% Sucesso & 14,3 & 30,0 & 100,0 & $p<0,045$ \\
\hline Incremento & $-0,16 \pm 0,71$ & $0,82 \pm 1,32$ & 1,91 & $p<0,19$ \\
\hline \multicolumn{5}{|l|}{ Geral } \\
\hline$N^{2}$ crianças & 19 & 28 & 23 & - \\
\hline \% Sucesso & 15,8 & 25,0 & 78,3 & $p<0,0001$ \\
\hline Incremento & $-0,18 \pm 0,67$ & $0,41 \pm 0,96$ & $1,71 \pm 1,31$ & $P<0,00001$ \\
\hline
\end{tabular}

- Comparação entre freqüência, casos de sucesso e médias de incremento de " $Z$ "

* Sucesso: Incremento de "Z" no indicador peso/idade superior a 0,55

42,34 para $10,1 \%$ ) na proporção de desnutrição global. Reduziu-se também a prevalência de formas agudas de desnutrição, que não mais existiam em outubro de 1991. As formas moderadas e graves de desnutrição (Gomez II e III), as quais afetavam a $33,8 \%$ das crianças em abril de 1989 , na última avaliação atingiam a $5,1 \%$ das mesmas. É visivel que a desnutrição global afetava predominantemente a aldeia Paranatinga na primeira avaliação (63,0\% contra 4,0\%). Em outubro de 1991 esta forma de desnutrição ainda acometia as crianças na aldeia Paranatinga, já em menor proporção. Observa-se que na aldeia Maroxewara a desnutrição sempre foi encontrada em formas leves e agudas, contrastando com a outra aldeia onde as formas crônicas, moderadas e graves foram mais freqüentes. Houve moderado aumento na prevalência de retardo de crescimento (desnutrição pregressa) nas duas aldeias.

Os valores dos indicadores antropométricos foma sistematicamente menores nas crianças da aldeia Paranatinga (Tabela 2), sendo que o índice peso/idade mostrou valores significantemente mais baixos entre as crianças do sexo feminino. Os valo- res dos indices guardavam relação com a idade, sendo menores nas crianças com mais de seis e menos de 24 meses de idade. Essas crianças foram também as que apresentaram em média maior incremento no indicador peso/idade. $\mathrm{Na}$ aldeia Maroxewara os índices não mostraram variação significativa em relação ao sexo e a idade, exceção feita à altura para a idade, que apresentava menores valores nas crianças mais velhas.

Nas duas aldeias a freqüência de casos de sucesso e $o$ incremento médio do indicador peso/idade foram tanto maior quanto mais severos eram os quadro de desnutrição (Tabela 3). Mesmo a média de incremento de crianças em desnutrição leve (Grau I) foi superior a das consideradas "notmais" (Geral: $\mathrm{H}=7,17, \mathrm{p}<0,008$ ). Para crianças em uma mesma classe nutricional, as diferenças entre as aldeias na freqüência de casos de sucesso e na média de incremento não foram significantes.

As medidas adotadas surtiram efeito rapidamente na aldeia Paranatinga, como mostra a Tabela 4. Em nove meses (abril de 89 a janeiro 91) observou-se nas crianças desnutridas aumento médio no indicador peso/idade superior ao estabelecido como 
Tabela 4. Quadro evolutivo do estado nutricional de 51 crianças desnutridas em abril de 89 , reavaliadas em janeiro de 90 e em outubro de $91^{*}$.

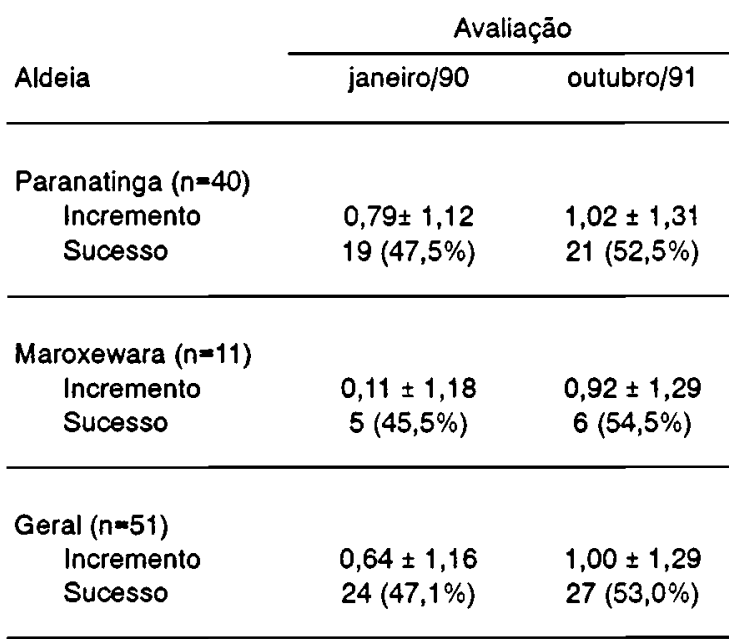

* Mesmas definições da Tabela 3.

Diferença entre as freqüèncias de casos de sucesso entre as médias de incremento das aldeias: $p>0,05$.

nível de sucesso; na aldeia Maroxewara o aumento foi mais pronunciado no periodo entre janeiro de 90 e outubro de 91 . Entretanto, para as crianças com algum grau de desnutrição, em nenhum momento foi significativa a diferença entre as aldeias quer na freqüência de casos de sucesso, quer no aumento médio do indicador.

Até 1989, a mortalidade na tribo se devia quase que exclusivamente às doenças infecciosas. A partir de então não ocorreu qualquer óbito por doenças diarréicas, infecções respiratórias, malária ou outra doença transmissivel. O aleitamento materno é uma prática corrente entre as mães parakanã, e prossegue por um período não inferior a 2 anos, não sendo conhecido um momento exato para o desmame, como norma social ou traço cultural desse povo.

\section{Discussão}

Os resultados obtidos de maneira geral corroboram o quadro clínico-epidemiológico observado; em nivel coletivo, retratam a recente evolução da realidade nutricional dos Parakanã. Assim é que a prevalência de desnutrição foi reduzida, e tem apresentado acentuada diferença entre as duas aldeias.

$\mathrm{O}$ aumento no número de crianças que apresentam retardo de crescimento após a melhoria do estado nutricional da população, e sua relação direta com a idade, mostrou ser uma seqüela decorrente da sobrevivência de crianças que estiveram severamente desnutridas, possivelmente pelo fato de terse logrado evitar o óbito, nesses casos, por doenças intercorrentes; semelhante efeito já foi relatado em outros estudos ${ }^{9,13,18}$.

A influência negativa do meio externo sobre o crescimento infantil, manifestada pela redução nos valores dos índices antropométricos, foi pronunciada nas crianças a partir do sexto mês de idade e, na aldeia Paranatinga, predominantemente no sexo feminino; este fato corrobora observações de outros autores, de que a partir desta idade o valo nutricional do leite materno é insuficiente para a manutenção de um adequado crescimento infantil ${ }^{13,18}$.

A melhora no estado nutricional obedeceu a padrões também já observados por outros autores, sendo tanto maior quanto mais severo era $o$ estado inicial da desnutrição ${ }^{9,13}$.

Apesar da suplementação alimentar ter sido importante para o desenvolvimento físico das crianças desnutridas, os dados do presente estudo não autorizam afirmar que ela tenha sido decisiva; no entanto, é possivel que na ausência de um aporte nutricional complementar as crianças desnutridas dificilmente teriam se desenvolvido da forma como se desenvolveram. O efeito do conjunto de medidas adotadas é que parece explicar a evolução observada do quadro nutricional, sendo a diferença na prevalência de desnutrição entre as aldeias atribuível às suas diferenças sociais e à historicidade de suas estruturas. Certas práticas culturais parakanã comprometem ainda mais o estado nutricional infantil ${ }^{14}$.

Os cultivos conhecidos pelos indios Parakanã (milho, mandioca e outros tubérculos) são praticados com métodos primitivos, de baixa produtividade, e sempre representaram complementação à dieta preferida por eles: a caça. A pesca, sazonal, não oferece maior aporte de proteínas à dieta da comunidade, principalmente na aldeia Paranatinga. $O$ consumo de frutos silvestres contribui com uma parcela mínima na alimentação desse povo ${ }^{15}$.

Nas duas aldeias, o alimento obtido (agricultu- 
ra, coleta, caça ou pesca), sempre foi dividido igualitariamente. No entanto, na última década, este comportamento modificou-se na aldeia Paranatinga, talvez pela escassez da caça (a aldeia está próxima do limites da reserva, perto da estrada) e pelo adensamento populacional. Nessa aldeia o modo de produção é desigual: alguns grupos familiares distribuem entre si a propriedade das roças que, por sua vez, não são proporcionais ao tamanho dos grupos. O mesmo ocorre com a distribuição inter e intrafamiliar de alimentos, que privilegia certas familias e o homem adulto em detrimento das mulheres e crianças. Na Maroxewara, a produção e a distribuição do alimento é natural e comunitária.

Os índios da aldeia Paranatinga estão há 20 anos em contato mais constante com membros de nossa cultura, por viverem perto da rodovia Transamazônica. Estão próximos de pontos de compra e venda de mercadorias, sendo comum desviarem seus esforços produtivos para culturas comerciáveis (banana, açaí, castanha-do-pará) em prejuizo das culturas de subsistência; como agravante, até início dos anos 90 era comum comercializarem os alimentos produzidos, que deixavam assim de ser consumidos pela própria população. $O$ dinheiro arrecadado nessas transações é utilizado na satisfação de necessidades criadas por novos hábitos socioculturais (alimentos manufaturados, cigarros, roupas e outros bens não produzidos pela comunidade) e parece representar uma nova forma de afirmação sociopolítica. Ao final do ano de 1988 , seus habitantes foram atingidos por uma epidemia de varicela; suas consequiências sobre o estado nutricional das crianças são previsiveis: perda metabólica importante de nitrogênio, anorexia e pais incapacitados para prover alimentos ${ }^{10,14}$. Apesar do Programa ter fornecido naquela época alimentação a praticamente todo os habitantes, tal medida, paliativa, dificilmente contornaria todos os efeitos negativos da infecção.

Por sua vez, os habitantes da aldeia Maroxewara, com menor tempo de contato, residem em área isolada da rede viária existente, com acesso por rios que somente são navegáveis durante a época das cheias (dezembro a março); os pequenos aviōes fretados são a única alternativa de transporte permanente, porém extremamente dispendiosa. A caça é farta e, em determinadas épocas, também o peixe. Enquanto nessa aldeia os homens fazem a farinha a partir da mandioca em maior quantidade, usando formos improvisados com tachos de zinco fornecidos pelo Programa, na Paranatinga ainda é atividade exclusivamente feminina, fazendo uso de rústicos tachos de barro, sendo menor a produção.

Ações coordenadas nas áreas de saúde, educação e estímulo à produção têm sido desenvolvidas, e suas conseqüências sobre a nutrição dos Parakanã não puderam ser dimensionadas isoladamente; a intervenção dos serviços de saúde sobre o estado nutricional infantil continua ocorrendo no sentido de recuperar a saúde dos menores desnutridos, mas a solução definitiva para esta questão depende do sucesso das ações acima mencionadas, particularmente as atividades de apoio à produção, que visam a tornar esta tribo auto-suficiente na produção de alimentos tanto para subsistência quanto para geração de excedentes e que, ao longo dos 25 anos de vigência do Programa Parakanã, permitirão a autosuficiência do povo Parakanã, preservando sua identidade enquanto nação indígena.

O sucesso de medidas de atenção primária em reverter a curto prazo quadro importante de desnutrição infantil instalado entre os Parakanã, mostra que a medicina preventiva é uma alternativa eficaz e factivel em melhorar o estado nutricional de populações humanas, particularmente em regiões remotas. É paradoxal que os grupos humanos mais vulneráveis via de regra não sejam contemplados com ações de saúde adequadas, necessárias para alcançarem nível de qualidade de vida aceitável. Enquanto não se solucionar este problema, não se logrará grau adequado de eqüidade. Neste contexto, os serviços de saúde, convenientemente estruturados e adaptados à realidade das comunidades às quais servern, desempenham uma função preponderante ao proporcionarem assistência primária à saúde com eficiência, eficácia e eqüidade ${ }^{3}$.

Entre os Parakanã já foram atingidas uma das metas propostas pela OMS (redução em $50 \%$ na prevalência de desnutrição na infância) no plano "Saúde para todos no ano $2000^{\prime \prime}$, mas este problema ainda preocupa, pois acomete uma população pequena e jovem, estando suas causas relacionadas a fatores primordialmente de ordem social, cuja superação depende de intevenções que não se encontram no âmbito de nossas relações inter-culturais, pelo que precisam ser dirigidas pelos próprios membros daquela comunidade. 
MARTINS, S.J \& MENEZES, R. C. [Nutritional status of Parakanã Indian children from birth through 5 years of age, eastern Amazonia, Brazil]. Rev.Saúde Pública, 28: 1-8, 1994. The evolution of the nutritional status of children under 5 years of age living in two Indian settlements of the Parakanã tribe, Maroxewara and Paratininga, situated in the southest of Pará State (Brazil), with less than 20 years of direct contact with our society, was studied. The main purpose of this study was to register the effects of the preventive and curative health activities of the "Parakanã Program" (created by an agreement between the National Indian Foundation- FUNAI and Northern Hydroelectric Project- ELETRONORTE), undertaken with the technical cooperation of the Tropical Medicine Center (Federal University of Pará). Anthropometric data were obtained in there cross-sectional studies (April 89; January 90 and October 91) for the purpose of evaluating the prevalence of malnutrition by means of Gomez's, Waterlow's and WHO criteria. The evolution of nutritional status was evaluated in the light of the rate of growth and accepting weight increments superior to those expected among well-nourished children as a goal. Seventy children $(87.5 \%$ of all the 0-5 years-olds living there) were followed through throughout the studies. Prevalence of malnutrition was greater in Paranatinga than in Maroxewara, possibly because the former was more populous and had had longer inter-racial contact. Paranatinga's anthropometric indices (wt/age, ht/age and wt/ht) were the lower. Most of the severe forms of malnutrition were found there too. Children between six months and 2 years old were the most affected. There had occurred an overall $76.1 \%$ decrease in the prevalence of malnutriotion. The primary health care given has been successful after eight months of activities, mainly in Paratininga. The increase of the wt/age index was proportional to the initial severity of the malnutrition various of the socio-cultural features observed might explain the differences in prevalences reported in this paper.

\section{Referèncias Bibliográficas}

1. ANJOS, L.A. Índices antropométricos e estado nutricional de escolares de baixa renda de um munícipio do Estado do Rio de Janeiro (Brasil): um estudo piloto. Rev. Saúde Pública, 23: 221-9, 1989.

2. BEGHIN, I. et al. A guide to nutritional assessment. Geneva, World Health Organization, 1988.

3. CERQUEIRA, M.T. Educación en nutrición: metas y metodologia, Bol. Of.Sanit.Panam., 99: 498-509, 1985.

4. DEAN, J. et al. EPI INFO Version 5. Atlanta. Center for Disease Control, Forecasting, and Impact Assessment Unit-WHO, 1989.

5. FAGUNDES-NETO, U.et al. Observations of the Alto Xingu indians (Central Brazil) with special reference to nutritional evaluation in children. Am.J.Clin.Nutr., 34: 2229-35, 1981.

6. FERREIRA, H.S. \& OTT, A.M.T. Avaliação do estado nutricional de crianças menores de cinco anos do Estado de
Rondônia, Brasil. Rev.Saúde Pública, 22: 179-83, 1988.

7. GRANT, J.P. The state of the world's children 1991. New York, UNICEF, 1991.

8. HORWITZ, A. El costo de la malnutrición. In: Organización Panamericana de la Salud. Vigilancia alimentaria y nutricional en las Americas. Washington, 1989 (OPS - Publicación Cientifica, 516).

9. LEI, D.M.L. et al. Medindo o impacto de programas de recuperação nutricional de pré-escolares: teste de uma metodologia. Rev.Saúde Pública, 23: 230-5, 1989.

10. MATA, L. Interacciones infeccion-nutricion. In: Cuminsky, M.; Moreno, E.M.; Suárez Ojeda, E.N. Crescimento y desarrollo- hechos y tendencias. Washington, Organización Panamericana de la Salud, 1988. p. 229-49 (OPSPublicación Cientifica, 510).

11. MOLINA, M.C.B. et al. Nutritional status of children of urban low-income communities, Brazil (1986). Rev. Saúde Pública, 23: 89-97, 1989.

12. MONTEIRO, C.A. Critérios antropométricos no diagnóstido da desnutrição em programas de assistência à criança. Rev.Saúde Pública, 18: 209-17, 1984.

13. MUSGROVE, P. Por uma mejor alimentación : evaluación de programas destinados a mejorar el consuno alimentario y el estado nutricional de familias pobres en Brasil. Washington, Organización Paramericana de la Salud, 1989. (OPS - Cuaderno Técnico, 25).

14. PROGRAMA PARAKANÃ - Convênio ELETRONORTE/FUNAI. Relatório do Sub-Programa de Saúde. Tucuruí, 1991. cap. 3: Estado nutricional da populaçào no periodo de maio de 1989 a setenibro de 1991. [mimeografado].

15. PROGRAMA PARAKANÃ-Convênio ELETRONORTE/FUNAI. Relatório de atividades do sub-programa de vigilância e produção - 1991. Tucuruí, 1991. [mimeografado].

16. PROGRAMA PARAKANÃ-Convênio ELETRONORTE/FUNAI. Infonmações, março de 1992. Tucurui, 1992. [mimeografado].

17. ROCHA, F.J \& SALZANO, F.M. Anthropometric studies in Brazilian Cayapo indians. Am.J.Phys.Anthropol., 36:95102, 1972.

18. SCHILLING, P.R. Supplementary feeding programs: a critical analysis. Rev.Saúde Pública, 24: 412-9, 1990.

19. SMITH, G.S. \& OLSON, J.G. Rapid epidemiologic assessment: evaluation of heal th problems and programs. $B O S$ TID Dev. 7: 16-9, 1987.

20. SOKAL, R.R. \& ROHLF, F.J. Biometria: principios y métodos estadísticos en la investigación biológica. Madrid, Ed. H. Blume, 1979.

21. VICTORA, C.G. et al. A saúde das crianças dos estados do Ceará, Rio Grande do Norte e Sergipe, Brasil: descrição de uma metodologia para diagnósticos comunitários. Rev.Saúde Pública, 25: 218-25, 1991.

22. WATERLOW, J.C. et al. The presentation and use of height and weight data for comparing the nutritional status of groups of children under the age of 10 years. Bull. World Heallh Org., 55: 489-98, 1977.

23. WHO WORKING GROUP. Use and interpretation of anthropometric indicators of nutritional status. Bull. World Health Org., 64: 929-41, 1986.

Recebido para publicação em 29.6.1992

Reapresentado em 13.5.1993

Aprovado para publicação em 14.12.1993 